\title{
Prior's Puzzle Generalized ${ }^{a}$
}

\author{
JUSTIN D'AMBROSIO \\ Australian National University
}

${ }^{a}$ Forthcoming in Philosophy and Phenomenological Research.

\begin{abstract}
Prior's puzzle is standardly taken to be the puzzle of why, given the assumption that that-clauses denote propositions, substitution of "the proposition that $P$ " for "that $P^{\prime \prime}$ within the complements of many propositional attitude verbs is invalid. I show that Prior's puzzle is much more general than is ordinarily supposed. There are two variants on the substitutional form of the puzzle-a quantificational variant and a pronominal variant-and all three forms of the puzzle arise in a wide range of verbal complements, rather than merely in the complements of propositional attitude verbs. The generalized puzzle shows that a range of proposed solutions to the original puzzle fail, or are radically incomplete, and also reveals the connections between Prior's puzzle and debates over the nature of semantic types and higher-order quantification. I go on to develop a novel, higher-order solution to the generalized form of the puzzle, and I argue that this higher-approach is superior to its first-order alternatives.
\end{abstract}

\section{Prior's Puzzle}

The standard theory of propositional attitude verbs is that they denote binary relations between agents and propositions. A sentence of the form "S Vs that $\mathrm{P}$ " is true iff the subject referred to by "S" stands in the relation V to the referent of the that-clause, which is a proposition. But if that-clauses refer to propositions, we should be able to substitute other, co-referring expressions for that-clauses salva veritate. ${ }^{1}$ But in many cases we cannot. Consider the following pair:

Contact: Justin D'Ambrosio <justin.d'ambrosio@anu.edu.au>

o. Thanks to Daniel Stoljar, Zoltán Gendler Szabó, Lloyd Humberstone, and Alan Hájek for many helpful comments, both in writing and in conversation, and to audiences at the Melbourne Logic Group and the Australian National University for their feedback.

1 . We should be able to make such substitutions on the assumption that the positions in which that-clauses occur are fully extensional. This is an assumption made by nearly everyone in the debate, and I will make it here, too. Also, throughout I will use the terms "refer" and 

a. Sally fears that Fido bites.
b. Sally fears the proposition that Fido bites.

The standard theory tells us that "that fido bites" refers to a proposition. It also seems clear that "the proposition that Fido bites" refers to a proposition-indeed, the very same proposition. And yet ( $1-a)$ can be true while ( $1-b)$ is false-Sally does not fear an abstract object. We may call this Prior's puzzle, after Arthur Prior [1971, p. 16], who first formulated it.

This paper first shows that Prior's puzzle is not just a puzzle concerning substitutions such as the one above. Rather, the failure of such substitutions to preserve truth is a special case of a puzzle that is much more general along two dimensions. First, the substitutional puzzle has both quantificational and pronominal variants that have the same source and warrant the same solution as the original form. Thus, Prior's puzzle has nothing specifically to do with substitution. Second, all three of these forms of the puzzle arise in the complements of all kinds of verbs. Thus, Prior's puzzle has nothing specifically to do with that-clauses or propositional attitude verbs.

This generalization has several important consequences. First, the generalized puzzle shows that a wide range of proposed solutions to Prior's puzzle fail, or are radically incomplete. Many such proposals apply only to the substitutional puzzle, and cannot be generalized to its quantificational or pronominal variants. Other proposals apply only to the case of propositional attitudes, but cannot be extended to solve the puzzle as it arises in other verbal complements. Still other views fail in both of these ways.

Second, this generalization reveals the way in which Prior's puzzle is deeply intertwined with debates over the nature of semantic types and the status of higher-order quantification. In particular, one's approach to solving the generalized form of Prior's puzzle is determined by, and also reveals, one's views on two questions that are fundamental to semantic theorizing: whether all expressions in a language refer to their semantic values, and relatedly, whether all quantifiers in natural language-including quantifiers that occur in predicate, sentence, and

\footnotetext{
"denote" interchangeably to pick out the single relation of interpretation used in standard compositional semantic theories. The key feature of this relation is that it is a two-place relation that takes names in both of its argument positions-standardly, the name of an expression and then the name of the object denoted by that expression-the object denoted typically being a set.
} 
adverbial positions, among others-can be reduced to first-order quantifiers over semantic values of the appropriate kinds.

There are two general approaches to semantics which answer these questions in opposing ways. The first approach is the standard one within compositional semantic theories that trace their origins to Frege via Montague. Such theories employ a single, first-order interpretation function, and reduce quantifiers in all grammatical positions to first-order quantifiers, thus answering the two questions in the affirmative. This assimilationist approach to semantics entails what I call the nominal assimilation approach to Prior's puzzle. The second approach is the one adopted by theorists who make use of higher-order resources in the semantic metalanguage. Such approaches employ a higher-order interpretation function, and higher-order resources in the metalanguage, and in doing so answer both questions in the negative. This higher-order, anti-assimilationist approach to semantics entails what I call the non-nominal resistance approach to Prior's puzzle.

I conclude the paper by contrasting representatives of these two strategies, and developing a novel, resistance solution to the generalized form of the puzzle. I first present the assimilationist view of Forbes [2006, 2018]. I show that while Forbes' view provides an intuitive solution to a wide range of instances of the generalized puzzle, assimilationism itself is subject to a range of objections. I then show how to develop a resistance solution by extending the semantic framework due to Williamson [2013], and show how it overcomes the objections to assimilationism. However, I go on to argue that the best form of the resistance approach incorporates the key insights of Forbes' proposal, while still making use of higher-order resources.

\section{Quantificational and Pronominal Variants of the Puzzle}

Prior's puzzle is typically presented as a puzzle concerning a distinctive kind of substitution within the complements of propositional attitude verbs-substitution of a propositional description such as "the proposition that $\mathrm{P}$ " for its embedded that-clause. It is also sometimes taken to concern the substitution of names for that-clauses, as in (2): 
(2) a. Gödel fears that mathematics reduces to logic.

b. Gödel fears logicism.

Nebel [2019]

Just as in (1). (2-a) does not entail (2-b), despite the fact that "logicism" and "that mathematics reduces to logic" appear to refer to the very same proposition. Call this form of substitution-in which a nominal expression is substituted for an apparently co-referential non-nominal one-nominal substitution. ${ }^{3}$ Prior's puzzle is ordinarily taken to be the puzzle of accounting for why nominal substitution for the complements of many propositional attitude verbs-construed as an inference or argument-is invalid.

But Prior's puzzle has nothing specifically to do with substitution. Consider again our example from above:

(3) Sally fears that Fido bites.

Suppose that the that-clause in (3) refers to a proposition. We can then generalize over that proposition; (4) follows from (3):

(4) Sally fears something.

But now consider the standard semantics for the existential quantifier in typetheory: 4

(5) $\llbracket \exists u_{x} \phi \rrbracket^{M, g}=1$ iff there is some $a \in D_{x}$ such that $\llbracket \phi \rrbracket^{M, g_{u_{x}}^{a}}=1$

In (5), $u_{x}$ is a variable of type $x$, and $\llbracket \phi \rrbracket^{M, g_{u_{x}}^{a}}$ is the result of assigning $a$ to occurrences of $u_{x}$ in $\phi$. Letting $\langle s, t\rangle$ be the type of object denoted by "that fido bites" - the type of propositions-the clause yields that (4) is true iff:

(6) There is some $a \in D_{\langle s, t\rangle}$ such that Sally fears $a$.

Clearly, (6) can be false even when (3) is true; Sally can fear that Fido bites

3. A note on terminology. By "nominal" expression I mean an expression of type $e$ that has a denotation within the domain of individuals, $D_{e}$. Such expressions are also sometimes called "referring expressions" or "singular terms". I will also use "nominal variable" for a variable of type $e$. This use of "nominal" picks out a semantic or a logical category, rather than a syntactic one. In English syntax, there are expressions headed by nouns-e.g. quantified noun phrases-that do not count as nominal by this definition.

4. See, for instance, Dowty et al. [1981. p. 91], van Benthem and Doets |1983], Enderton 2001], Väänänen [2019], among many others. 
without there being some $a$ in the set of propositions such that Sally fears $a$. Thus, on the assumption that the standard semantics for the existential quantifier is correct, the apparently valid inference from (3) to (4) turns out to be invalid. We can make a similar point in the object language. Consider (7), which is the result of translating (6) into the object language:

(7) Some proposition is such that Sally fears it.

On the assumption that that-clauses refer to propositions, (7) should be equivalent to (4), and so should follow from (3). But clearly it does not follow from (3). and so is not equivalent to (4).

Call the form of quantification whose truth-conditions are given by (5) nominal quantification, and call quantifiers whose semantics are given by (5) nominal quantifiers. The key feature of nominal quantification is that it spells out the truth-conditions of quantified sentences such as (4) in the metalanguage using first-order quantification over sets. Since first-order quantifiers bind variables in name positions, the clause in (5) assimilates quantification over the entire clausal complement of "fears" to quantification into a name position. This is why nominal quantification warrants its name. In what follows, I will use "nominal quantification" and "first-order quantification" interchangeably.

The examples above show that nominal, or first-order, existential generalization over the semantic values of the complements of propositional attitude verbs is invalid. Moreover, given the interdefinability of the existential and universal quantifiers, we can expect that nominal generalizations of all kinds over the semantic values of such verbal complements will be invalid. Further, both nominal substitution and quantification yield the same kind of absurdity; both yield the result that Sally fears an abstract object-a proposition—although (1-b) makes clear just which proposition she fears.

We can formulate another variant of the puzzle using pronouns. Consider the following example:

(8) a. Sally fears that Fido bites.

b. Sally fears it.

c. Sally fears so.

Here, instead of functioning like a bound variable, as in (7), "it" behaves like a 
free variable, and the standard way of interpreting such variables is as receiving their semantic values from the assignment function. But even if we stipulate that the assignment function assigns to "it" the very proposition denoted by "that Fido bites", the meaning of (8-b) differs from that of (8-a) in exactly same way we observed in the substitution and quantificational puzzles: as in (7). (8-b) is interpreted as meaning that Sally fears an abstract object, and so does not follow from (8-a). This contrasts with (8-c), which preserves (8-a)'s natural reading.

Now consider a dialogue in which (9-b) follows (9-a):

(9) a. Sally fears that Fido bites.

b. *Bill fears it, too.

c. Bill fears so, too.

Call the anaphoric use of "it" in (9-b) a case of nominal anaphora. In the dialogue, (9-b) is awkward at best; nominal anaphora is infelicitous. By contrast, (9-c) uttered on the heels of (9-a) is fully felicitous. These cases show that Prior's puzzle arises for pronouns both when they serve as substituends, and when they are used anaphorically in discourse. While I think the anaphoric form of the puzzle is important and distinct from the substitutional form, going forward I will for the most part set infelicity and anaphora aside, and consider only truth preservation. As a consequence, going forward I will focus on pronouns only as they figure into the substitutional form of the puzzle.

The substitutional and quantificational forms of the puzzle are intimately related. If nominal quantification into a position is valid, then substitution of singular terms-including names, definite descriptions, and appropriately interpreted pronouns-will likewise be valid, because these singular terms serve as witnesses for nominal existential quantifiers. ${ }^{6}$ Conversely, if one can validly substitute nominal expressions into a particular position, then nominal existential quantification into that position will be valid, because the referents of those nominal expressions will be the objects over which nominal existential quantifiers generalize, given the standard semantics. Thus, the validity of nominal substitution and nominal quantification go hand in hand. ${ }^{7}$

6. By "witness" I here mean an expression that, when substituted for the existential quantifier, yields a true substitution instance of the quantified sentence.

7. The validity of nominal substitution and quantification likewise go hand in hand with the felicity of nominal anaphora, but as I said, I will here set this issue aside. 
This connection between nominal substitution and nominal quantification is well known. At least since Quine [1956] and Kaplan [1968], we have known that nominal quantification and the substitution of co-referential nominal expressions, including pronouns, fail in concert within the complements of attitude verbs, propositional or otherwise. This gives us reason to think that the failures of nominal substitution and generalization in Prior's have the same source, and that jointly, such failures form a unified, more general puzzle. Moreover, given that nominal substitution and quantification have traditionally been used to test expressions for whether they are referring expressions, we have good reason-at least preliminarily - to think that the failure of both can be traced to the assimilation of expressions whose semantic function is not to refer to expressions whose function is to refer.

\section{Generalization to other Grammatical Positions}

Not only does Prior's puzzle have both quantificational and pronominal variants, but neither the original puzzle nor its variants are specific to that-clauses or propositional attitude verbs. Rather, perfectly analogous puzzles arise in a variety of other positions. Consider the following examples:

(10) a. Sally seeks a unicorn

b. Sally seeks the generalized quantifier denoted by "a unicorn".

(ii) a. Sally forgot who came to the party.

b. Sally forgot the question of who came to the party. ${ }^{8}$

(12) a. Sally became wise.

b. Sally became the property of being wise. ${ }^{9}$

8. You might think that this case does not differ from the propositional case, because to forget who came to the party is to forget that $x, y$, and $z$ came to the party. This would be a mistake. First, the same phenomenon occurs for verbs such as "investigate" that are not reducible to propositional attitudes (see Groenendijk and Stokhof [1984], Lahiri] [2002], George [2011], Friedman 2013]). Second, even if embedded questions are true just in case the embedding verb relates you to an answer to that question, wh-clauses still denote questions-i.e. sets of answers-and so we should be able to pick out that same answer with a nominal expression without a change of truth-value. But clearly we cannot. The same phenomenon arises for many other verbs, including "study", "investigate", "overlook", "see", "know", etc.

9. Friederike Moltmann 2003. 2004 uses this example to illustrate what she calls the 
On the traditional, Montagovian semantics for the notional reading of an intensional transitive verb, the intensional NP "a unicorn" denotes an intensional generalized quantifier. ${ }^{10}$ But substitution of a description of this semantic value in (10) changes the sentence's truth-conditions-(10-a) can be true while (10-b) is false. Similarly, substitution of a description of the question denoted by the wh-phrase in (11) changes the sentence's truth-conditions: Sally can forget who came to the party without forgetting a set of propositions. In (11), while Sally might have become wise, she did not become the property of being wise.

Further, the quantificational form of the puzzle arises for (10-a) (23-a) as well. Consider the following inferences:

(13) a. Sally seeks a unicorn.

b. Sally seeks something.

(14) a. Sally forgot who came to the party.

b. Sally forgot something.

(15) a. Sally became wise.

b. Sally became something.

In each case, application of the standard semantics for the existential yields the following truth-conditions for (13-b) (15-b), respectively: ${ }^{12}$

(16) There is some $a \in D_{\langle s,\langle\langle s,\langle e, t\rangle\rangle, t\rangle\rangle}$ such that Sally seeks $a$.

(17) There is some $a \in D_{\langle s,\langle s, t\rangle\rangle}$ such that Sally forgot $a$.

(18) There is some $a \in D_{\langle s,\langle e, t\rangle\rangle}$ such that Sally became $a$.

objectivization effect. All of the examples of invalid substitutions here are instances of the objectivization effect.

10. On Montague's view, such quantifiers are in fact properties of properties, so we might also make use of the alternative substituend "the property of properties denoted by 'a unicorn' ". This proposal is not uncontroversial. On another account of the notional reading, due to Zimmermann 1993, 2006a], intensional NPs denote properties. But as we will see below, the same problem arises for this proposal.

12. Here, and throughout, I make use of what I take to be the standard type-assignments for expressions of the relevant kind within a functional type theory, like the one developed by Montague [1974]. Nothing in my arguments turns on exactly how one types the relevant expressions, nor does anything turn on making use of functional as opposed to relational types-the two approaches to type theory are provably equivalent. 
If these standard truth-conditions for the existential quantifier are correct, then each of the generalizations in (13) (15) are invalid. Each of (16) (25) can fail to be satisfied even while (13-a) (24-a) are true. ${ }^{13}$ We observe the same phenomenon with the object-language counterparts of (16) (18).

(19) Some property of properties is such that Sally seeks it.

(20) Some set of propositions is such that Sally forgot it.

(21) Some property is such that Sally became it.

These sentences do not follow from (13-a) (15-a), and so if they are equivalent to the generalizations in $\left(13^{-b}\right)(15-b)$, then the existential generalizations are invalid. Further, as expected, the pronominal variant of the puzzle arises in all of these cases again as well. If "it" in each of (19) (21) were to occur unbound, and be assigned the very same semantic values as the complements of (13) (15). respectively, the result would not preserve truth. This contrasts with the use of non-nominal proforms such as "one", "that", and "thus", substitution of which do preserve truth. Thus, Prior's puzzle does not merely concern substitution, but rather likewise concerns both nominal quantification and the interpretation of pronouns, and arises in a wide range of verbal complements. In all of these positions, the puzzle appears to arise from nominal assimilation: from the assimilation of expressions whose function is not to refer to referring expressions, and the assimilation of quantifiers that bind higher-type variables to first-order, nominal quantifiers.

One final point is in order concerning the range of positions in which Prior's puzzle arises. It may seem that Prior's puzzle even has instances that arise outside of verbal complements. Moltmann [2003] and Zimmermann [2006b] discuss what appear to be instances of Prior's puzzle that arise in subject position, as in

13. Moltmann 2003, 2004, 2008] calls quantifiers such as the ones in (13) (24) "special quantifiers". While she points out that such quantifiers are distinctive, she does not point out that treating them as nominal quantifiers renders basic quantificational inferences invalid. And while her views on the semantics of such quantifiers have changed, one proposal is that such quantifiers range over so-called "variable objects". But this view, even if intelligible, likewise renders these inferences invalid. Zimmermann 2006a) offers an account of special quantifiers as higher-order quantifiers, but interprets higher-order quantification in a set-theoretic framework, and as a consequence fails to solve the quantificational puzzle. I discuss Zimmermann's view in the next section. 
(22):

a. Ordinariness is boring.

b. The property of being ordinary is boring.

Zimmermann 2006b

Here we can suppose that 'ordinariness' denotes the property of being ordinary, and so that the subjects of (22-a) and (22-b) corefer. Nonetheless, ordinariness can be boring while the property of being ordinary is not- $(22-\mathrm{a})$ can be true while (22-b) is false. This is an apparent instance of Prior's puzzle. Further, consider (23):

(23) a. Sally painted carefully.

b. Sally painted the property of events denoted by "carefully".

(24) a. Sally painted carefully.

b. Sally painted somehow.

(25) There is some $a \in D_{\langle\langle e, t\rangle,\langle e, t\rangle\rangle}$ such that Sally painted $a$.

Here again, we seem to observe the same behaviours as above. Surely Sally can paint carefully without painting a function from properties to properties, which again indicates that $\left(25^{-a}\right)$ can be true while $\left(25^{-b}\right)$ is false.

These purported instances of Prior's puzzle differ in important ways from the instances that arise for verbal complements, and seem to be much more obviously defective. First, following Zimmermann [2006b], one might think that (22). unlike (22-b), is not a singular predication at all, but triggers a rule other than functional application. This would differentiate (22) from the previous examples, in which the complement of the verb denotes an argument of the verbal predicate. The same, one might think, is true for (23) and its quantified variants: unlike the verbal complements in (10) (12). "carefully" does not even purport to denote one of the verb's arguments, but instead denotes a modifier. Thus it seems that in these cases, the logical form of the sentence has changed in a way that it has not in our original examples. ${ }^{15}$ Going forward I will officially exclude these kinds of cases from the remit of Prior's puzzle, construing Prior's puzzle as con-

15. I am grateful to an anonymous reviewer for help with this point. Below, in developing a solution to Prior's puzzle, it will become clear what these examples have in common with the instances of the puzzle that arise within the complements of verbs. 
cerning verbal complements. However, the solution to Prior's puzzle that I will develop below treats it as a consequence of a perfectly general issue concerning inter-categorial substitution, and this solution will reveal the underlying similarities between failures of truth-preservation within verbal complements and such failures in subject, adjunct, and other positions.

The result is that Prior's puzzle can be generalized along two dimensions in a way that yields a new, unified, and more significant puzzle. First, Prior's puzzle arises when three first-order operations that standardly preserve truthsubstitution of co-referential constant expression, first-order existential generalization, and substition of an appropriately interpreted pronoun-fail when applied in the complement of a verb. These operations are intimately related in that they jointly make up the interconnected machinery of first-order quantification theory. If substitution of a particular nominal expression or an appropriately interpreted pronoun preserves truth, then so will nominal existential generalization, because the denotation of that substitution instance guarantees the truth of the existential quantification, given the standard semantics. Likewise, if nominal existential quantification over a verbal complement is valid, then nominal substitution will likewise be valid, for such substituends are the witnesses for first-order quantifiers. Thus these these operations stand or fall together.

Second, while Prior's puzzle has typically been thought to arise only for propositional complements, it in fact arises for all manner of verbal complements, and moreover it seems to arise for the same reason in each case. The complements in which the puzzle arises are standardly taken to denote sets from a type domain other than that of type $e-$ from higher type domains. The puzzle arises when we attempt to name, nominally quantify over, or pronominally refer to the higher-order semantic value of that complement. In other words, it arises whenever we nominalize a higher-order complement by means of applying one of the three first-order operations just mentioned. Together, these two dimensions of generalization show that Prior's puzzle concerns not only substitution in the complements of propositional attitude verbs, but rather our entire apparatus of reference and quantification in the complements of all manner of different verbs.

This generalization of the puzzle has an immediate negative result: it reveals that a wide a range of proposed solutions to the substitutional form of Prior's puzzle are radically incomplete-and fail to solve the generalized puzzle-or 
perhaps fail altogether. Appreciating how such proposals fail will reveal the kind of solution that stands to succeed, and so it is to these failures that I now turn.

\section{Criticism of Existing Proposals}

The first casualties of the generalized puzzle are views such as those endorsed by Zimmermann [2006b], Grzankowski [2018], and Nebel [2019], on which the invalidity of nominal substitution is explained by the fact that nominal substituends denote different things than the expressions they replace. First, consider Nebel's view. On his view, "the proposition that Fido bites" does not refer to a proposition. Rather, it denotes a propositional concept-a function from worlds to propositions. Thus, on Nebel's view, (1-a) can be true while (1-b) is false because they have different internal arguments. This allows Nebel to preserve the view that that-clauses refer to propositions, while accounting for the change in truthvalue. It also allows him to maintain that propositional attitude verbs are univo-

cal across substitutions such as (25) - the only difference between (1-a) and (1-b) lies in their arguments.

However, Nebel's proposal fails when it confronts the quantificational and pronominal forms of the puzzle. To see this, we can first ask: does Nebel's view validate nominal EG? More specifically, does Nebel's view validate the inference from (1-a) to (4), where (4) has the semantics in (6)?

(1-a) Sally fears that Fido bites.

(4) Sally fears something.

(6) There is some $a \in D_{\langle s, t\rangle}$ such that Sally fears $a$.

Or, equivalently in the object language, does (1-a) entail (7)?

(7) Some proposition is such that Sally fears it.

Given that Nebel holds that that-clauses refer to propositions, his answer should be "yes"; nominal EG over non-empty referring expressions in extensional contexts appears to be an incontrovertible logical principle. But if Nebel answers "yes", then he fails to solve the quantificational puzzle; as we saw above, the 
quantificational puzzle just is the puzzle of showing why nominal quantification into such positions is invalid. So in order to solve the puzzle Nebel must answer "no". In fact, Nebel [2019, p. 92] seems to endorse a negative answer when he claims that "proposition", as it occurs in sentences such as (26), does not denote a set of propositions:

(26) Sally fears a proposition.

Rather, on his view, "proposition" denotes a set of propositional concepts, and so (26) is true iff Sally fears a particular member of that set. Thus, he claims, (7) does not follow from (1-a). But this reasoning entails that nominal existential generalization is invalid, which contradicts the assumption that "that Fido bites" is a referring expression in the first place. The only way out is for Nebel to hold that that-clauses refer to propositions, that nominal EG over those clauses is invalid, and to develop a non-nominal semantics for "something" that validates the inference from (1-a) to (4)-a seemingly impossible position to maintain. ${ }^{17}$

But this is not the last of Nebel's problems. Nebel's view (a) fails to solve the pronominal form of the puzzle; (b) is specific to the propositional case; and (c) contrary to what he claims, does not allow propositional attitude verbs to remain fully univocal, since propositional attitude verbs would have to be type-shifted in order to accept propositional concepts as arguments. I conclude that his view fails.

Similar problems doom Zimmermann's [2006b] view. Consider (27) and (28):

(27) John seeks a unicorn.

(28) John seeks the property of being a unicorn.

Clearly, (28) does not follow from (27). On Zimmermann's view of ITVs, "a unicorn" in (27) denotes a property: $\lambda x \lambda w\left[\right.$ unicorn' $\left.^{\prime}(w)(x)\right]$. By contrast, "the property of being a unicorn" denotes a distinct, higher-order property: roughly,

17. Even if Nebel claims that "proposition" denotes a set of propositional concepts, and $D_{p}$ does not denote the set of propositions, then EG with the following semantics must be valid on his view:

(i) There is some $a \in D_{p}$ such that Sally fears $\vee a$,

where ${ }^{\vee} a$ is the extension of the propositional concept $a$-namely, a proposition. But it clearly is not. 
the property of being the property of being a unicorn. Thus, he claims, (28) predictably does follow from (27), because they denote different properties. But this view likewise fails to solve the quantificational puzzle. To see this, we can ask whether (29) follows from (27):

(29) John seeks something.

If "a unicorn" refers to a property, then the inference from (27) to (29) should be valid when "something" has the semantics in (5), adapted to the type of properties. But on this semantics, (29) is equivalent to (30):

(30) Some property is such that John seeks it,

which does not follow from (27). Thus Zimmermann's proposal fails to solve the quantificational form of the puzzle for the same reasons as Nebel's.

The generalization of the puzzle to the non-propositional case also undermines another strategy for addressing Prior's puzzle-a strategy adopted by Moffett [2003], Parsons [1993], and Harman [2003]. They hold that solving Prior's puzzle is simply a matter of finding the appropriate kinds of objects for each attitude, and finding the appropriate nominal substituends to pick out these objects. For example, while we may not fear propositions, we do plausibly fear states of affairs; while we may not know propositions, we do know facts; and while we do not want propositions, we do want various outcomes. Once we find the appropriate substituends, they argue, nominal substiution and quantification are valid.

Moffett 2003] adopts a strategy of this sort to solve the problem of "doxastic shift", which is, in effect, Prior's puzzle applied to "knows". The problem is that, while one can know that $\mathrm{P}$, it does not follow that one knows the proposition that $\mathrm{P}$; rather, in the sense relevant here, one knows facts, not propositions. Accordingly, Moffett proposes a change in the rule governing predication for verbs like "know". On his view, while that-clauses denote propositions, the predicate "is known by Sally" applies to facts corresponding to propositions, as opposed to propositions themselves. Parsons [1993 develops a similar view on which in some cases, that-clauses denote propositions, while in others they denote facts that are "determined" by those propositions. Harman [2003], likewise, holds that in cases where we observe the substitution failure, that-clauses must denote 
objects distinct from, but suitably related to propositions.

But again, this approach does not solve the generalized puzzle; it fails when we consider the generalization of the puzzle to other grammatical categories. The approach fails in the case of intensional transitive verbs, as in (10), in the case of questions, as in (11), and in the case of adverbs, as in (23). The problem in these cases is that any nominal subsitution will yield an invalidity. Once we recognize this, we see that Moffett's view forces (10-a) to be false-an unacceptable conclusion. ${ }^{19}$ Parsons' and Harman's views fails for the same reason: in many cases, there are no truth-preserving nominal substituends to be found. These failures show that attempting to solve the generalized puzzle by finding the appropriate kind of object for each attitude is a lost cause.

\section{Resistance and Assimilation}

The generalized form of Prior's puzzle can be thought of as a test for whether an expression serves to refer to its semantic value. If an expression refers to its semantic value, then substitution of co-referential nominal expressions will preserve truth, and nominal EG over that expression's referent will be valid. When co-referential nominal substitutions for an expression do not preserve truth, and when nominal EG is invalid, it tells us that the expression does not serve to refer, but instead serves a different semantic function. ${ }^{20}$ Call such expressions non-nominal expressions, and call the positions in which they occur non-nominal positions. ${ }^{21}$ A solution to the generalized form of Prior's puzzle requires us to explain why nominal substitution and quantification into non-nominal positions is invalid. It also requires us to develop a theory that vindicates valid generalizations into such positions, such as the generalization from (1-a) to (4). This latter task requires us to either develop a theory of non-nominal quantification, or to show how nominal quantification into such positions can be valid after all.

19. For a different criticism of Moffett's view, see Forbes [2018.

20. This argument is only valid on the supposition that the substitution does not change the interpretation of the verb. Denying this supposition is in fact one major route of response, which I will discuss presently. Note also that here substitutivity and nominal quantification are treated as necessary conditions on being a referring expression; they may not be sufficient conditions for counting as a referring expression.

21. As with my use of "nominal expression", I use "nominal position" to pick out a semantic or logical position, rather than a syntactic one. 
There are two general strategies for meeting these desiderata. The first is what I call the strategy of nominal assimilation. This strategy aims to solve Prior's puzzle in two steps. The first step is to assimilate all non-nominal expressions to nominal ones, and all non-nominal quantifiers to nominal, first-order quantifiers. In undertaking this joint assimilation, the assimilationist treats every expression of the object language as, in effect, a proper name of its semantic value, and because as first-order quantifiers are quantifiers binding name positions, the assimilationist treats all expressions of the object-language as open to such quantification. $^{22}$ The second step in solving Prior's puzzle is then to use one of a variety of mechanisms to account for why nominal substitution fails to preserve truth, and to show how nominal quantification is valid after all. The most plausible of these mechanisms-which we discussed above, and to which we will return below-is to hold either that nominal substitution and quantification occasion a change in the meaning of the verb, and so explain the Priorian invalidities in terms of equivocation.

Assimilationism is entailed by, and standardly developed within, the approach to semantics that can be traced to Frege via Montague-what I call the assimilationist approach to semantics. The key feature of the assimilationist approach to semantics is that it employs a single, first-order relation of semantic interpretation that relates expressions of all different types in a language to objects of appropriate kinds. This relation is what I have here been calling "reference" or "denotation". Standardly, the objects in the range of the interpretation relation are objects drawn from appropriate subsets of the domain of a model, and indeed, model-theoretic semantics is assimilationism's dominant form. Since model-theoretic semantics treats the semantic values of all object-language expressions as elements of a set-the domain of a model-and set theory is a firstorder theory, model-theoretic semantics is a fundamentally first-order approach to semantics. The standard approach to quantification in model-theoretic semantics, as in assimilationist approaches more generally, is to adopt the nominal semantics for quantification given in (5), which treats quantification of different orders as many-sorted first-order quantification over appropriate subsets of a domain.

22. As $\overline{\text { Potts }}[1979 \mid$ points out the dominant view of the $\lambda$-calculus since Church [1951] has been that each $\lambda$-expression serves as a proper name. 
The assimilationist approach to semantics is closely related to what Prior [1971. Ch. 3] himself called the program of Platonism. The basic idea behind the program of Platonism is to convert every expression-perhaps excepting logical and copular expressions-to a referring expression while preserving meaning. Such meaning preservation is possible, Prior claims, provided one is willing to engage in a program of paraphrase or regimentation. Lewis, a proponent of assimilationism, makes just this point in discussing the possibility of replacing a language's theoretical terms with nominally quantified variables:

We may stipulate that our $T$-terms are names, not predicates or functors. No generality is lost, since names can purport to name entities of any kind: individuals, species, states, properties, substances, magnitudes, classes, relations, or what not. Instead of a $T$-predicate ' $F_{-}$', for instance, we can use '_- has F-hood'; 'F-hood' is a T-name purporting to name a property, and '__ has __' is an $O$-predicate. It is automatic to reformulate all $T$-terms as names, under the safe assumption that our $O$-vocabulary provides the needed copulas:

'_- has the property _-'

'__ is in the state __ at time _-'

'__ has _- to degree __'

and the like. We will later replace the T-terms with bound variables; by making the $T$-terms grammatically uniform, we avoid the need to introduce variables of diverse types. [Lewis, 1970]

Here Lewis illustrates the assimilationist strategy perfectly: we can assimilate all expressions to names so long as we are prepared to regiment those sentences to accommodate nominalization. Other proponents of the assimilationist strategy are King [2002], Forbes [2006, 2018], both of whom will be discussed below.

The second strategy for solving Prior's puzzle is what I call the strategy of non-nominal resistance. This strategy aims to solve Prior's puzzle by maintaining that non-nominal expressions are genuinely semantically different from nominal ones, and insisting that non-nominal quantifiers cannot be reduced to first-order quantifiers ranging over different subsets of the first-order domain. Rather, on the resistance proposal, expressions of different semantic types-for example, 
predicates, quantifiers, modifiers, and various kinds of verbal complements-do not serve to name their semantic values, but rather have a variety of different semantic functions; predicates predicate, quantifiers quantify, modifiers modify, etc. Further, quantifiers that replace expressions of these different types are irreducibly higher-order; such quantifiers are not nominal quantifiers. As a result, substitution between these categories is illegitimate, and Prior's puzzle is the result of illicitly assimilating expressions with one semantic function to expressions with another-it is the result of illicit nominalization. With this account of what has gone wrong with nominal substitution and quantification, the resistance view then aims to come up with a theory of non-nominal quantification that validates the requisite quantificational inferences.

This approach to Prior's puzzle is entailed by, and is naturally developed within, the approach to semantics that makes use of fundamentally higher-order resources in the metalanguage-what I call the resistance approach to semantics. The resistance approach is motivated by a range of semantic, logical, and ontological considerations, many of which stem from a dissatisfaction with assimilationism. ${ }^{25}$ Among the resistance is Prior himself. ${ }^{26}$ Prior held that only names refer, and so there is no question of what non-nominal expressions designate or refer to, for designation is not in their semantic job description. Further, Prior held that quantification into non-nominal positions is intelligible on its own terms, and should not be reduced to first-order quantification over semantic values. On this view, the generalized form of Prior's puzzle is the result of illicitly treating positions in which expressions with higher semantic types occur as name positions, and reducing quantifiers that generalize into such positions to first-order ones.

The key difference between the assimilationist and resistance programs lies in their approach to semantic interpretation. On assimilationist approaches, semantic interpretation is a first-order binary relation between expressions of a

25. Making use of higher-order resources in the metalanguage is thought to (i) provide remission from various ontological commitments [Yablo. 1996. Rayo and Yablo, 2001], (ii) allow us quantify over absolutely everything |Williamson, 2003. Linnebo, 2006. Rayo and Uzquiano. 2006], (iii) allow us to state a fully general semantic theory for a language [Linnebo and Rayo. 2012. Williamson, 2013], and to solve the concept horse problem |Jones, 2016]. Wright [2001] and Liebesman |2015| develop resistance views that attempt to solve the concept horse problem, but neither makes use of higher-order resources in the metalanguage.

26. See in particular Prior [1971. pp. 32-41]. 
language and entities in a model, expressed in the metalanguage by a first-order binary relation symbol. Thus, the only semantic distinctions between different expressions in a language come from the fact that they bear this relation to different kinds of objects, and quantifiers into different grammatical positions are distinguished only in ranging over different subsets of the first-order domain. By contrast, the resistance strategy employs a mechanism of semantic interpretation that is itself higher-order-i.e. the resistance approach employs a higher-order interpretation or assignment function in the metalanguage. The semantics for higher-order quantification can then be specified in terms of this higher-order interpretation function, which allows the quantifiers to be genuinely higher-order, and avoids first-order reduction. The next section explores how the assimilationist and the resistance strategies attempt to solve the generalized puzzle.

\section{Two Representatives Compared}

\subsection{Nominal Assimilation: Forbes}

Recall our examples of the substitutional puzzle from above.

(10) a. Sally seeks a unicorn.

b. Sally seeks the generalized quantifier denoted by "a unicorn".

(1i) a. Sally forgot who came to the party.

b. Sally forgot the question of who came to the party.

(12) a. Sally became wise.

b. Sally became the property of being wise.

For the moment, focus only on (10-b) (12-b). What is the right semantics for these sentences? Intuitively, (10-b) and (11-b) involve ordinary transitive verbs, and have direct objects that are denoted by the definite descriptions that serve as substituends. (12-b) seems to involve an identity reading of "become". Yes, these sentences are strange, but we have an intuitive grasp of their meanings: each of them expresses a relation between Sally and an abstract object. Moreover, there is strong intuitive pull to the idea that the nominal substituends in (10) (23) denote the same things as the expressions they replace; "the generalized quantifier 
denoted by 'a unicorn' " appears to denote the same thing as "a unicorn", and likewise for the other pairs. That's why the (b)-sentences seem so strange-they seem to say that the subject bears a relation to an abstract object that they would normally only bear to something concrete.

These considerations support the view that what changes in each of these substitutions is not the semantic value of the verb's complement, but rather the denotation of the verb itself. Views of this kind are developed by King 2002 and Forbes [2018], although both focus only on the case of propositional attitude verbs. King and Forbes argue that that-clauses and their corresponding propositional descriptions refer to the same things, but that-clauses and their corresponding propositional descriptions differ syntactically, and it is this syntactic difference that occasions the change in the verbal denotation. Here I will focus primarily on Forbes' view, but much of what I say straightforwardly carries over to King's view as well. ${ }^{28}$

As Forbes [2018] develops this view, substitution of a noun phrase for a coreferring that-clause changes the thematic role played by the propositional denotation of both expressions. On this view, Prior's puzzle is the result of a shift in the argument structure of the verb that results from a change in the syntax of its complement. Returning to our original example, on Forbes' view, (31-a) has the logical form in (31-b), while (32-a) has the logical form in (32-b):

$$
\begin{aligned}
& \text { a. Sally fears that Fido bites } \\
& \text { b. } \exists e[\text { fear }(e) \& \operatorname{in}(e, \text { Sally }) \& \operatorname{content}(e, \text { that Fido bites })]
\end{aligned}
$$

a. Sally fears the proposition that Fido bites

b. $\exists e[$ fear $(e) \&$ in $(e$, Sally $) \&$ theme(e,the proposition that Fido bites $)]^{29}$

In (31), Sally is in a state of fearing, and "that fido bites" refers to a proposition that serves as the content of that state. By contrast, in (32), Sally is in a state of fearing, and "the proposition that Fido bites" refers to a proposition that serves as the theme of that state-i.e. the state's direct object. Thus, Forbes' view pre-

28. King's view and Forbes' view differ in that King's is classical, while Forbes' is neoDavidsonian. In virtue of being neo-Davidsonian, Forbes view has a few slight advantages, to which I will return in the conclusion.

29. Forbes derives these logical forms compositionally using a sequent calculus, together with a type theory that takes events as a basic type. Here I present only the logical forms, but the type-theoretic background will become relevant below. 
serves the view that both that-clauses and their corresponding descriptions refer to propositions, but takes the syntactic difference between phrasal and clausal complements to occasion a change in the role played by that proposition. The exact same view can be extended to solve the problem that arises from substitution of a pronoun such as "it" for the that-clause complement. This solves the substitutional form of Prior's puzzle

What about the quantificational form? Consider the inference from (31-a) to (33):

(33) Sally fears something.

Is this inference valid? If we combine Forbes' semantics with the standard clause for the quantifier in (5), the result is (34):

$$
\exists a \in D_{p} \exists e[\text { fear }(e) \& \text { in }(e, \text { Sally) \& } \operatorname{content}(e, a)]
$$

This clearly follows from (31-a), when (31-a) is given the semantics in (31-b). Thus, by complicating the semantics of the attitude ascription, Forbes' view vindicates nominal EG into the complement of "fears".

Finally, why did nominal quantification into the complement of "fears" originally look invalid? The reason is that "fears" is ambiguous between thematic and non-thematic versions, and quantification into the theme role clearly does not follow from (31-a) Moreover, truth conditions like (35) and paraphrases such as (36) force the thematic reading of the verb:

(35) There is some $a \in D_{p}$ such that Sally fears $a$.

(36) Some proposition is such that Sally fears it.

On Forbes' view, both of these sentences have the following semantics:

$$
\exists a \in D_{p} \exists e[\text { fear }(e) \& \text { in }(e, \text { Sally }) \& \text { theme }(e, a)]
$$

Since (37) involves a different argument structure than $(31-b)$, the inference from (31-a) to (37) is not valid. This explanation of the invalidity of nominal quantification thus pairs perfectly with the explanation of the invalidity of nominal substitution. Both are invalid because they force the verb to have its direct-object, "thematic" reading. 
Moreover, while Forbes' focuses only on propositional attitudes, his view can be generalized to a wide range of cases. For example, Forbes can tell an exactly parallel story for intensional transitive verbs such as "seeks". On Forbes' view, sentences such as (10-a) have the following semantics:

a. Sally seeks a unicorn.

b. $\exists e[\operatorname{search}(e) \& \operatorname{agent}(e, \operatorname{Sally}) \& \operatorname{char}(e, Q)]$

Where $Q$ is the generalized quantifier denoted by "a unicorn". We can then give the semantics of $(39-a)$ as in $(39-b)$ :

(39) a. Sally seeks something.

b. $\exists a \in D_{q}, e[\operatorname{search}(e) \& \operatorname{agent}(e, \operatorname{Sally}) \& \operatorname{char}(e, a)]$,

where $D_{q}$ is the domain of generalized quantifiers. On this semantics, (39-a) clearly follows from (38-a) via nominal existential generalization. Moreover, this semantics makes sense of the role that abstract objects play in the semantics of intensional verbs. Substitution of a nominal expression for "a unicorn" serves to change the role that the denotation of "a unicorn" plays in the event:

(40) a. Sally seeks the generalized quantifier denoted by "a unicorn".

b. $\exists e[\operatorname{search}(e) \& \operatorname{agent}(e$, Sally) \& theme(e,the generalized quantifier denoted by "a unicorn")]

In (4o-b), the substitution of a definite for an indefinite has occasioned a change in the argument structure of the verb; it specifies the theme of the verb, rather than characterizing it. Similar extensions of Forbes' framework account for the rest, or at least the majority of, the examples above.

Forbes' view assimilates apparently non-nominal positions-that-clauses, intensional NP complements, question complements, etc-to referring expressions; each such expression refers to a set of an appropriate type. Further, by complicating the semantics of the verbs in question, and positing an ambiguity, it renders nominal quantification valid on one of these readings: propositions frequently serve as the contents of states of fearing, but rarely as their themes. The distinction between content and theme is a highly intuitive component of Forbes' solution.

However, Forbes' view faces a problem. One of the key features of the view is 
that "the proposition that Fido bites" and "that Fido bites" co-refer-his view is an assimilationist view. But on Forbes' view, which is developed within type theory, these expressions also have different semantic types. These facts entail that Forbes' view confronts a version of the concept horse problem. To see this, first note that the two expressions differ syntactically, and in virtue of this syntactic difference force different argument structures for the verb "fear"—one involving a "theme" role and another involving the "content" role. The theme of a state of fearing is of type $e$, while the content is of type $t$ (or of type $\langle s, t\rangle$ in an intensional setting). Thus, on Forbes' view, "that Fido bites" denotes a truth-value, and "the proposition that Fido bites" denotes an entity. But on the plausible assumption that no members of $D_{t}$ are members of $D_{e}$, Forbes' view leads to a contradiction, for it entails an identity between something of type $t$ and something of type $e$.

A closely related problem arises when we try to specify the denotations of expressions such as "the property of being a unicorn", as in (41):

(41) "The property of being a horse" denotes a function from entities to truthvalues.

In Forbes' type-theory, (41) is true because "the property of being a horse" is exactly what property-type expressions like "is a unicorn" denote. But "the property of being a unicorn" is a referring expression, and so denotes an object in $D_{e}$. But then, by (41), some member of $D_{e}$ is a function from entities to truth-values. So some member of $D_{e}$ is a member of $D_{\langle e, t\rangle}$. But this cannot be, since no function is a member of its own domain. The very same problem arises if we assign "the property of being a horse" another type, such as $\langle\langle e, t\rangle, t\rangle \cdot .^{31}$

Moreover, similar worries arise whenever we have expressions of type $e$ that pick out objects other than properties-i.e. expressions such as "the proposition that Fido bites", "the generalized quantifier denoted by 'a unicorn"', and "the property of being a unicorn", "Logicism", etc, in our language. If we assign a type to these expressions, then the denotations of such expressions must be in $D_{e}$. But when we try to specify their semantics, we will often be able to show that their denotations are both in $D_{e}$ and in some higher type domain generated from

31. Here I am grateful to Zoltán Gendler Szabó, who offers a very similar objection to type theory in his talk "Semantic Categories". Szabó himself rejects type theory altogether in favor of an account which employs a plural notion of semantic interpretation. I am sympathetic to his view, but will not discuss it further here. 
type $e$. But on the assumption that denotations are sets, the axiom of regularity guarantees that this is impossible. ${ }^{32}$

One potential strategy for solving these problems comes from Cresswell [1973]. Cresswell maintains that higher type domains are not disjoint from the nominal domain; on his view, everything there is can be named. ${ }^{34}$ As a consequence, he [1973, p. 99] takes every thing of every type to be a subset of the nominal domain, $\mathbf{D}_{1}$. But given that no function can be in its own domain, Cresswell is forced to treat functional types as interpreted with partial, as opposed to total functions. He treats the functions as undefined for every argument that would lead to a violation of the axiom of regularity. But this approach comes with costs. As Cresswell [1973, p. 100] admits, it yields the result that a range of sentences in the object-language have no semantic values at all, which is a severe expressive limitation, and even threatens bivalence.

Another, more direct response to the problem is to limit the applicability of the type theory, and to exclude expressions like "a function from entities to truth values" and other pieces of the semantic metalanguage from the interpreted fragment. But this likewise significantly restricts the expressive power of the theory, and artificially excludes a range of expressions to which a fully general theory would apply. Moreover, in adhering to orthodox type theory, we also encounter a range of other expressibility problems. Orthodox type theory cannot, for instance, express absolutely general quantification, and cannot provide a fully general semantics for a first-order language. These problems give us reason to look for a view that solves these problems. As I will argue below, such a view can be developed by retaining the neo-Davidsonian components of Forbes' proposal while giving up its assimilationist commitments.

\subsection{Non-Nominal Resistance: A Williamsonian Proposal}

The alternative to the strategy of nominal assimilation is to deny that that-clauses refer to propositions, and likewise to deny that quantifiers that replace that-

32. Assuming, of course, that the higher types are ones generated by type $e$. The proof is simple using the axiom of regularity. Suppose there is some function that is a member of its own transitive closure. Then there is some sequence of sets $x_{1} \ldots x_{n}$ such that $x_{n} \in x_{1} \in \ldots \in$ $x_{n-1} \in x_{n}$. But if we consider the set $a=\left\{x_{1} \ldots x_{n}\right\}$, we see that it is a violation of the axiom of regularity, since none of the members of $a$ are disjoint from $a$ itself.

34. For another view which denies that higher-type domains are disjoint from the nominal domain, see Chierchia [1984], Chierchia and Turner [1988]. 
clauses are nominal quantifiers over propositions. This strategy can then be generalized to all expressions for which Prior's puzzle arises. This is what I call the strategy of non-nominal resistance.

The strategy of non-nominal resistance is most naturally undertaken in a semantic framework that makes use of higher-order metalinguistic resources. 35 The key feature of higher-order semantic theories that allow them to solve the puzzle is that such theories generally employ higher-order mechanisms of semantic interpretation, and as a consequence, on such views, it is false that thatclauses refer, and a fortiori, false that they refer to propositions. While the materials for such a solution to Prior's puzzle are readily available in the literatureparticularly in the work of Rayo and Yablo [2001], Rayo [2006], Linnebo [2006], and Williamson [2013], and Krämer [2014 — no one has used these materials to formulate such a solution. Here I illustrate how to develop such a solution by extending the semantic ideas proposed by Williamson [2013].

Williamson, like higher-orderists generally, holds that expressions of different semantic categories have different semantic types. But instead of accounting for differences in semantic type by assigning each expression a set from the appropriate type domain, and so treating them as names of those objects, Williamson interprets expressions of different semantic types using a higherorder function in the metalanguage that serves as both an interpretation and assignment function. In the relational type theory in which Williamson presents his view, this function, $\underline{\mathrm{a}}_{\langle e, \lambda\rangle}$, has the type $\langle e, \lambda\rangle$ where $\lambda$ is the limit type which collects together all of the finite types. In a functional type theory, this expression would have type $\langle e,\langle\lambda, t\rangle\rangle$. Since I have so far been making use of functional types, in what follows I will convert Williamson's type assigments to functional types, although nothing of substance turns on this choice. ${ }^{36}$

Roughly speaking, assigning $\underline{\mathrm{a}}_{\langle e,\langle\lambda, t\rangle\rangle}$ the limit type in its second argument

35. Although it need not be. On the view proposed by Rieppel |2016], predicates (e.g. "happy") and their corresponding definite descriptions (e.g. "the property of being happy") bear different first-order semantic relations to one and the same denotation. However, it is unclear how Rieppel's view generalizes to cases other than that of predicates.

36. The relational type $\langle e, \lambda\rangle$ is ordinarily interpreted as a subset of $D_{e} \times D_{\lambda}$, which is exactly how the type $\langle e,\langle\lambda, t\rangle\rangle$ is interpreted-this latter type takes an element of $D_{e}$ and returns a characteristic function on $D_{\lambda}$ that yields true for each member of $D_{\lambda}$ to which the member of $D_{e}$ is related. Of course, the higher-order view disavows both of these interpretations, since both assign sets as the references of expressions of all types. 
position allows $\underline{\mathrm{a}}_{\langle e,\langle\lambda, t\rangle\rangle}$ to be radically type-polymorphic in its second argument position; expressions of type $\lambda$ are always also of some more specific finite type. This allows $\underline{\mathrm{a}}\langle e,\langle\lambda, t\rangle\rangle$ to simultaneously interpret expressions of each semantic type in the object language in a non-nominal, higher-order way appropriate to that type. Notably, $\underline{\mathrm{a}}_{\langle e,\langle\lambda, t\rangle\rangle}$ is only nominal in its second argument place when it is used to interpret expressions of type $e .37$

To understand how this approach to semantic interpretation bears on Prior's puzzle, consider the substitutional puzzle in the propositional case:

(1) a. Sally fears that Fido bites.

b. Sally fears the proposition that Fido bites.

Here, let us suppose that "that Fido bites" is of type $\langle s, t\rangle$. In that case, $\underline{\mathrm{a}}_{\langle e,\langle\lambda, t\rangle\rangle}$ applied to "that Fido bites" yields (42):

(42) $\underline{\mathrm{a}}_{\langle e,\langle\lambda, t\rangle\rangle}$ ("that Fido bites", that Fido bites).

In (42), the occurrence of "that Fido bites" in the metalanguage is not a referring expression, but retains non-nominal status-the second argument-place of the function $\underline{a}_{\langle e,\langle\lambda, t\rangle\rangle}$, in this instance, has not only the infinite type $\lambda$, but also the more specific finite type $\langle s, t\rangle$. In other words, $\underline{\text { a }}$ has propositional type in its second argument position, and so is appropriate for interpreting constants and variables of that type. It does not treat that-clauses as referring to propositions.

By contrast, application of $\underline{a}_{\langle e,\langle\lambda, t\rangle\rangle}$ to "the proposition that Fido bites" yields (43):

(43) $\underline{\mathrm{a}}_{\langle e,\langle\lambda, t\rangle\rangle}$ ("the proposition that Fido bites", the proposition that Fido bites), where the use of "the proposition that Fido bites" is of type e. Thus, the higherorder view interprets "that Fido bites" and "the proposition that Fido bites" differently-they are of different semantic categories, and thus have different interpretations. Given this difference, substituting the latter for the former is

37. Assuming that there is a limit type and employing it in the metalanguage is controversial. Krämer |2017], for example, argues that assuming cumulative types prevents us from expressing absolutely general quantification. However, nothing I say here turns on the assumption of such a type. Everything I say could be formulated using an alternative framework, such as the one presented by Krämer 2014, who proposes a hierarchy of denotation functors, $\operatorname{den}_{1} \ldots \operatorname{den}_{n}$, for each finite type $n$. 
not predicted to preserve truth. Since semantic types are not merely differentiated by denoting objects from different subsets of the first-order domain, the higher-order theorist denies that "the proposition that Fido bites" and "that Fido bites" refer to the same thing, and indeed that the latter refers at all. Thus the higher-order view predicts that substitutions such as (1) will not preserve truth.

On the contrary, given the semantic types of each of the expressions, the default prediction in cases of inter-type substitution is semantic uninterpretability. For the higher-order theorist, such substitutions are on a par with substituting a name for a predicate in first-order logic. On the higher order view, such substitutions are, quite literally, category mistakes, and so are predicted to be semantically ill-formed. This prediction is borne out by the large range of cases in which such substitutions do yield nonsense. For instance, consider the following pair:

a. Sally hopes that Fido is friendly.

b. Sally hopes the proposition that Fido is friendly.

Here, substitution of a propositional description for a that-clause yields ill-formedness. Such ill-formedness is the subject of another closely related puzzle called Rundle's puzzle, of which (44) is one instance. ${ }^{39}$ Rundle's puzzle, like Prior's puzzle, has both substitutional and quantificational forms, and occurs in a wide range of grammatical positions. But unlike Prior's puzzle, it concerns interpretability or grammaticality, rather than truth-preservation. For instance, when we try to substitute a nominal expression for a verb, for an attributive adjective, or for any number of other expressions, or when we try to nominally quantify into such positions, the result is uninterpretable, just as in (44)-one cannot hope a proposition. On the whole, the result of nominal substitutions is more often nonsense than sense; uninterpretability is the normal result. The higher-order view predicts exactly this.

The higher-order approach solves Prior's puzzle by treating it as a special case of Rundle's puzzle. The higher-order approach prohibits nominal substitution and nominal quantification on general, type-theoretic grounds. But sometimes-as in all of the cases of Prior's puzzle above-the result of such nominal substitution is well-formed and interpretable due to the presence of an

39. See Rundle |1967], Nebel [2019], and Author |forthcoming| for discussion of Rundle's puzzle. 
altogether different verb-in this case, transitive "fears" - that accepts expressions of type $e$ in its second argument position. On the higher-order view, while the default expectation for such intercategorial substitutions is ill-formedness, in some cases, verbs with complements of type $\langle s, t\rangle$ also have transitive forms whose complements are of type $e$-i.e. that accept the nominalizations of expressions of type $\langle s, t\rangle$. Such substitutions, while well-formed, are predicted to be generally invalid, because both the verbs and their complements have different types, and so different interpretations. Thus, the higher-order view solves both Rundle's puzzle and Prior's puzzle in one fell swoop.

The higher-order view also solves the quantification puzzle easily. To see this, consider again (3) and (4)

(3) Sally fears that Fido bites.

(4) Sally fears something.

On the higher-order view, "something", as it occurs in (44), is an irreducibly higher-order quantifier that binds a variable of type $\langle s, t\rangle$. Thus, (4) has the form given in (45):

$$
\left.\exists v_{\langle s, t\rangle}\left[\text { fears(Sally, } v_{\langle s, t\rangle}\right\rangle\right]
$$

Modifying Williamson's view slightly, we can give the semantics of (45) as follows, where both $\underline{\mathrm{a}}$ and $\underline{\mathrm{b}}$ are of type $\langle e,\langle\lambda, t\rangle\rangle$ as we saw above:

$$
\begin{aligned}
& \operatorname{TRUE}\left(\left\ulcorner\exists v_{\langle s, t\rangle}\left(\text { fears(Sally, } v_{\langle s, t\rangle}\right\urcorner\right), \underline{\mathrm{a}}\right) \text { iff }(\exists \underline{\mathrm{b}} \text { (VARIANT(a, } \\
& \left.\left.\left.\underline{\mathrm{b}},\left\ulcorner v_{\langle s, t\rangle}\right\urcorner\right)\right) \wedge \operatorname{TRUE}\left(\left\ulcorner\text { fears(Sally, } v_{\langle s, t\rangle}\right\urcorner\right), \underline{\mathrm{b}}\right) .^{{ }^{1}}
\end{aligned}
$$

Roughly, (46) says that (45) is true on a just in case there is some higher-order assignment $\underline{b}$ that differs from $\underline{a}$ only in what it assigns to $v_{\langle s, t\rangle}$ and the open formula "Sally fears $v_{\langle s, t\rangle}$ " is true relative to that assignment. This clause is thoroughly higher-order: it makes use of a higher-order assignment function, higher-order quantification over that assignment function, and a higher-order relation, VARIANT, between two assignment functions and a variable.

This proposal simultaneously shows why inferences like the one from (3) to

41. Here I have converted the definition given by Williamson [2013. p. 238] to the existential case, omitted world variables from the metalanguage, and dropped the type-subscripts from $\underline{\text { a }}$ and $\underline{b}$ for readability. 
(4) are valid, and why construing "something" as a nominal quantifier renders the inference invalid. First, on this proposal, (4) true just in case (47) is true relative to some assignment of an appropriate value to the variable:

(47) fears(Sally, $\left.v_{\langle s, t\rangle}\right)$

Clearly, the assignment in (42) is just such an assignment, so on the semantics for "something" given in (46), the inference from (3) to (4) is valid. Thus, we have a theory of non-nominal quantification that does not reduce such quantifiers to many-sorted first-order quantifiers.

Second, this semantics reveals why nominal existential generalization is not predicted to be valid. On a nominal semantics, (4) is true just in case (47) is satisfied when $v_{\langle s, t\rangle}$ is assigned a member of $D_{p}$-i.e. when the assignment function assigns the variable some proposition. But the clause in (46) requires nothing of the sort. Since the members of $D_{p}$ are of type $e$, nominal quantification assigns $v_{\langle s, t\rangle}$ something of the wrong type, and so is predicted not to follow from (3) -it assigns the free variable in (47) a value of the wrong type. Thus, the higherorder view meets all three of the desiderata laid out above: it invalidates nominal substitution, invalidates nominal quantification, and validates non-nominal quantification. It also generalizes to every grammatical position for which the puzzle arises-the semantics above works for variables of any type-and solves the pronominal puzzle: pronouns can be treated as variables of different types.

This view also explains the invalidity of nominal substitutions and quantification in subject and adjunct positions, which above we officially excluded from the remit of Prior's puzzle, but which are in many ways similar to it. As in the case of substitution for and quantification over verbal complements, such failures to preserve truth arise because of illicit nominalization of an expression whose semantic function is not to refer. As with substitutions for verbal complements, the higher-order approach again prohibits such substitutions on general, type-theoretic grounds. The semantic function of a modifier is not to refer, and so nominal expressions such as "the property of events denoted by 'carefully' " do not refer to the same things as adverbs such as "carefully", because the latter expression does not refer at all. Thus such substitutions are not predicted to be truth-preserving.

Finally, the higher-order view also does not confront the version of the con- 
cept horse problem confronted by Forbes. On the Williamsonian view, it is false that "that Fido bites" refers to a proposition, and false that "the property of being a horse" refers to a function from objects to truth-values. What is true is (42); the claim that "that Fido bites" refers to a proposition is a loose, first-order paraphrase of this higher-order claim. Further, given that $\underline{a}_{\langle e,\langle\lambda, t\rangle\rangle}$ interprets "that Fido bites" and "the proposition that Fido bites" differently, no claim can be made that they co-denote or co-refer. The concept horse problem is the result of treating all expressions as referring expressions. Since, on the higher-order proposal, expressions of different semantic categories have different semantic functions, and only those of type $e$ can be said to refer, no such problem arises. ${ }^{42}$

However, the Williamsonian proposal does not address is the general fact that some inter-categorial substitutions are valid. For instance, for verbs like "believe" and "concede", nominal substitution of a propositional description for a that-clause does seem to be truth-preserving:

a. Sally believes that Fido bites.

b. Sally believes the proposition that Fido bites.

(49) a. Sally conceded that Fido bites

b. Sally conceded the proposition that Fido bites.

Given that the higher-order interpretation function interprets these expressions as having different semantic types, it appears to be a mystery why such substitutions are truth-preserving. Moreover, "believe" and "concede" are far from the only verbs that behaves this way.

However, the fact that such substitutions are valid appears to be a lexical fact about "believe" and verbs of that kind. The lexical fact is that their clausal and transitive versions are equivalent after nominal substitution. Here the higherorder view can take a cue from Forbes, and state meaning postulates connecting the transitive and intransitive versions of these verbs. For instance, the higherorder view can state the following postulate governing the two readings of "believe":

42. However, see Jones 2016 for an argument that higher-order interpretation is in fact a form of reference. Here I presume, as Williamson does, that there are different forms or modes of semantic interpretation for each different semantic category, and that only expressions of type $e$ refer. 
(50) believe $\langle e,\langle\langle s, t\rangle, t\rangle\rangle\left(\right.$ sally $_{e}$, that Fido bites $\left.{ }_{\langle s, t\rangle}\right) \leftrightarrow$ believe $_{\langle e,\langle e, t\rangle\rangle}\left(\right.$ sally $_{e}$, the proposition that Fido bites $e_{e}$

This makes the validity of nominal substitution for the complement of "believes" idiosyncratic to the meaning of "believes" itself. We might then also have to state meaning postulates for every other verb that licenses nominal substitution. This method, while somewhat ad hoc, is not implausible. It seems likely that the reason that deducing that $\mathrm{P}$ and deducing the proposition that $\mathrm{P}$ are equivalent has to do with the lexical meaning of "deduce", rather than any general principle governing verbs of this kind.

Here one might worry that, even if this approach provides a solution to Prior's puzzle, this solution isn't novel. Aren't there other higher-order, resistance approaches to quantification and attitude verbs that provide, or would provide, equally good solutions? What makes this view distinctive? There are two points to be made in response. First, it is true that there has been some recent work on higher-order approaches to propositional attitude ascriptions. In particular, Trueman 2018] has developed a resistance approach to the semantics of propositional attitude verbs, and Jones [2019] has developed a higher-order approach to the metaphysics of propositional attitudes themselves. It is likewise true that each of these proposals is at least suggestive of the kind of solution to Prior's puzzle that I have offered here. But neither Trueman nor Jones discuss Prior's puzzle in any of its forms directly, neither provides a semantics for non-nominal quantification, and neither addresses cases other than the case of propositional attitude verbs. Thus, while there is a clear consonance between those views and the view developed here, they merely hint at a solution to one aspect of a highly general semantic puzzle.

Second, there has been one attempt to provide a semantics for non-nominal quantification that validates the inferences at issue in Prior's puzzle: the proposal developed by Tobias Rosefeldt [2008]. While view claims to accomplish the same things as the view I have developed here, it does not do so, and is ultimately not a non-nominal semantics at all. To see why, let us consider the semantics Rosefeldt proposes for the existential quantifier. This semantics employs an interpretation function $I$, which is a function from expressions of a typed language into a model-it takes an expression of type $a$ to an object in the 
appropriate type domain $D_{a}$. Rosefeldt then defines what he calls $\alpha_{a}$-variant of an interpretation $I$. According to Rosefeldt, if $\alpha_{a}$ is a constant symbol of syntactic type $a$ in a language $L$, then

(51) an interpretation $I^{*}$ is an $\alpha_{a}$-variant of $I$ iff $I^{*}$ differs only in which element of $D_{a}$ it assigns to $\alpha_{a}$. Rosefeldt 2008, p. 322]

He then gives the truth-conditions for the existential quantifier as follows:

(52) If $\phi$ is of the form $\left\ulcorner\exists \chi_{a} \psi\right\urcorner$ then $\phi$ is true under $I$ iff $\psi\left[\alpha_{a} / \chi_{a}\right]$ is true under at least one $\alpha_{a}$-variant of $I$.

Rosefeldt 2008, p. 323]

The problem with this proposal is that $I$ is a function from expressions to objects of the appropriate type, and it is nominal in its output or value position. Thus, $I$ assigns a value to $\alpha_{a}$ from the appropriate domain by either naming that value or by nominally quantifying over objects from that domain. Thus, $I$ is simply a standard assimilationist interpretation function, and accordingly once again makes it the case that all expressions of the language are, in effect, proper names of their semantic values, and so incurs all of the problems outlined above for assimilationism. A genuinely non-nominal semantics requires a mechanism of interpretation that is higher-order-i.e. non-nominal-in its second argument position, of exactly the sort involved in the proposal above.

\section{Conclusion: A Neo-Davidsonian Resistance Solution}

As we have seen, the higher order approach offers a general solution to both Prior's puzzle and Rundle's puzzle by treating the former as a special case of the latter. However, Forbes' neo-Davidsonian approach is highly intuitive, and has a range of other benefits. Perhaps most importantly, since on Forbes' view, the only thing that changes in nominal substitution is the thematic role occupied by an argument, there is an important sense in which the verbs in questiontransitive and intransitive "fear", for instance-remain univocal. Both forms involve the unary property of events, fear(e). By contrast, on non-Davidsonian classical views, transitive and clausal "fear" simply express different relations that bear no interesting semantic relation to one another, but are, in certain cases, 
held together by a meaning postulate.

This provides the neo-Davidsonian view with a number of advantages. One is that it explains the semantic commonality between transitive and clausal versions of the verbs involved in Prior's puzzle. Second, and relatedly, this allows the neo-Davidsonian view to avoid arguments raised by Nebel [2019] to the effect that coordination of clausal and transitive versions of "fear" give rise to zeugma. The Davidsonian view predicts that they will not. Finally, the Davidsonian view is metaphysically perspicuous-it does not simply treat transitive and clausal versions of a verb as different relations, but rather makes clear what differs about each of the states denoted by those events-they have different argument structures.

Luckily, the distinction between classical and neo-Davidsonian approaches to Prior's puzzle cuts across the distinction between assimilationist and resistance strategies. It is perfectly possible to make use of a neo-Davidsonian theory at the first-order level, and make use of higher-order resources in the metalanguage. That is to say, we can opt for a resistance form of Forbes' neo-Davidsonian proposal. The only change that would be required is to relinquish the idea that "the proposition that Fido bites" and "that Fido bites" co-refer. We can then spell out the semantics for the neo-Davidsonian type theory using irreducibly higherorder resources, in ways that will allow us to avoid the problems with orthodox type theory, and also allow us to make use of the generality of the higher-order view that allows us to solve Rundle's puzzle. The result is an attractive package of views on which we introduce and preserve a distinction between an attitude verb having a theme, and it having content, but avoid the view that expressions of higher semantic types function semantically in the same way as their nominalizations.

\section{References}

Author. Redacted. forthcoming.

Gennaro Chierchia. Topics in the Syntax and Semantics of Gerunds and Infinitives. PhD thesis, UMass Amherst, 1984 .

Gennaro Chierchia and Raymond Turner. Semantics and property theory. Linguistics and Philosophy, 11:261-302, 1988.

Alonzo Church. A formulation of the logic of sense and denotation. In H. M. Kallen P. Henle 
and S. Langer, editors, Structure, Method, and Meaning, pages 3-24. The Liberal Arts Press, New York, 1951.

Max Cresswell. Logics and Languages. Methuen and Co. Limited, London, 1973.

David Dowty, Robert E. Wall, and Stanley Peters. Introduction to Montague Semantics. D. Reidel Publishing Company, 1981.

Herbert B. Enderton. A Mathematical Introduction to Logic. Hardcourt Academic Press, 2nd edition, 2001.

Graeme Forbes. Attitude Problems. Oxford University Press, Oxford, 2006.

Graeme Forbes. Content and theme in attitude ascriptions. In A. Grzankowski and M. Montague, editors, Non-Propositional Intentionality. Oxford University Press, Oxford, 2018.

Jane Friedman. Question-directed attitudes. Philosophical Perspectives, 27(1):145-174, 2013.

B. R. George. Question Embedding and the Semantics of Answers. PhD thesis, University of California, Los Angeles, Los Angeles, 2011.

Jeroen Groenendijk and Martin Stokhof. Studies on the Semantics of Questions and the Pragmatics of Answers. PhD thesis, Universiteit van Amsterdam, 1984.

Alex Grzankowski. A relational theory of non-propositional attitudes. In Alex Grzankowski and Michelle Montague, editors, Non-Propositional Intentionality. Oxford University Press, 2018.

Gilbert Harman. Category mistakes in m\&e. Philosophical Perspectives, 17(1):165-180, 2003.

Nicholas Jones. A higher-order solution to the problem of the concept Horse. Ergo, 3(6):132-166, 2016.

Nicholas K. Jones. Propositions and cognitive relations. Proceedings of the Aristotelian Society, CXIX(Part 2):157-178, 2019.

David Kaplan. Quantifying in. Synthese, 19(1/2):178-214, December 1968.

Jeffrey C. King. Designating propositions. Philosophical Review, 111(3), 2002.

Stephan Krämer. Semantic values in higher-order semantics. Philosophical Studies, 168:709-724, 2014 .

Stephan Krämer. Everything, and then some. Mind, 126(502):499-528, 2017.

Utpal Lahiri. Questions and Answers in Embedded Contexts. Oxford University Press, Oxford and New York, 2002.

David K. Lewis. How to define theoretical terms. The Journal of Philosophy, 67(13):427-446, 1970.

David Liebesman. Predication as ascription. Mind, 124(494):517-569, 2015.

Oystein Linnebo. Sets, properties, and unrestricted quantification. In Agustín Rayo and Gabriel Uzquiano, editors, Absolute Generality, pages 149-178. Clarendon Press, Oxford, 2006.

Oystein Linnebo and Agustín Rayo. Hierarchies ontological and ideological. Mind, 121:269-308, 2012.

Marc A. Moffett. Knowing facts and believing propositions: A solution to the problem of doxastic shift. Philosophical Studies, 115(1):81-97, July 2003.

Friederike Moltmann. Nominalizing quantifiers. Journal of Philosophical Logic, 32:445-481, 2003.

Friederike Moltmann. Nonreferential complements, nominalizations, and derived objects. Journal of Semantics, 21:1-43, 2004.

Friederike Moltmann. Intensional verbs and their intentional objects. Natural Language Semantics, 16:239-270, 2008.

Richard Montague. Formal Philosophy: Selected Papers of Richard Montague. Yale University Press, 
1974 .

Jacob M. Nebel. Hopes, Fears, and other grammatical scarecrows. Philosophical Review, 2019.

Terence Parsons. On denoting propositions and facts. Philosophical Perspectives, 7 (Language and Logic):441-460, 1993 .

Timothy C. Potts. 'the grossest confusion possible?' - frege and the lambda-calculus. Revue Internationale de Philosophie, 33(130):761-785, 1979.

A. N. Prior. Objects of Thought. Oxford University Press, New York and Oxford, 1971.

W.V. Quine. Quantifiers and propositional attitudes. The Journal of Philosophy, 53(5):177-187, March 1956.

Agustín Rayo. Beyond plurals. In Agustín Rayo and Gabriel Uzquiano, editors, Absolute Generality, pages 220-254. Clarendon Press, Oxford, 2006.

Agustín Rayo and Gabriel Uzquiano, editors. Absolute Generality. Clarendon Press, Oxford, 2006.

Agustín Rayo and Stephen Yablo. Nominalism through de-nominalization. Nô̂s, 35(1):74-92, 2001.

Michael Rieppel. Being something: Properties and predicative quantification. Mind, 125(499), 2016.

Tobias Rosefeldt. 'that'-clauses and non-nominal quantification. Philosophical Studies, 137: 301-333, 2008.

B. Rundle. Transitivity and indirect speech. Proceedings of the Aristotelian Society, 68(1967-1968): 187-206, 1967.

Robert Trueman. The prenective view of propositional content. Synthese, 195:1799-1825, 2018.

Jouko Väänänen. Second-order and higher-order logic. In Edward N. Zalta, editor, The Stanford Encyclopedia of Philosophy. Fall 2019.

Johan van Benthem and Kees Doets. Higher-order logic. In D. Gabbay and F. Guenther, editors, Handbook of Philosophical Logic, volume 164 of Synthese Library (Studies in Epistemology, Logic, Methodology, and Philosophy of Science). Springer, Dordrecht, 1983.

Timothy Williamson. Everything. Philosophical Perspectives, 17:415-465, 2003.

Timothy Williamson. Modal Logic as Metaphysics. Oxford University Press, Oxford, 2013.

Crispin Wright. Why frege does not deserve his grain of salt. In Bob Hale and Crispin Wright, editors, The Reason's Proper Study: Essays Toward a Neo-Fregean Philosophy of Mathematics, chapter 3. Oxford University Press, 2001.

Stephen Yablo. How in the world? Philosophical Topics, 24(1):255-286, Spring 1996.

Thomas Ede Zimmermann. On the proper treatment of opacity in certain verbs. Natural Language Semantics, 1:149-179, 1993.

Thomas Ede Zimmermann. Monotonicity in opaque verbs. Linguistics and Philosophy, 29: 715-761, December 2006a.

Thomas Ede Zimmermann. The values of semantics. In Patrick Brandt and Eric Fuß, editors, Form, Structure, and Grammar, pages 383-397. Akademie Verlag, Berlin, 2006b. 